\title{
Combining Time-Resolved Deformation Experiments with Electron Tomography to Understand Dislocation-Particle Interactions
}

\author{
G.S. Liu,* I.M. Robertson* \\ * Dept. of Materials Science and Engineering, U. Illinois, 1304 W. Green Street, Urbana IL 61801.
}

Strengthening mechanisms in metals involve increasing the density or strength of barriers to mobile dislocations. In the case of particle strengthened systems, strengthening at room temperature is controlled by the particle coherency and size and is achieved through the dislocation either cutting through or bowing around the particle. The latter mechanism leaves a loop and occurs for incoherent and large coherent particles. Computer simulations [1,2] and time-resolved TEM straining experiments $[3,4]$ suggest a multitude of interactions rather than the few depicted in textbooks, which generate dislocation loops in association with the interaction. At higher temperatures, dislocation climb adds another bypass process. Here again, a greater variety of interactions are observed than have been envisioned from simple dislocation processes. In order to advance the development of predictive models of strengthening and creep, it is necessary to understand the parameter that dominates the dislocation-particle interaction process and how it changes with increasing strain. Although past in situ TEM straining experiments have yielded some insight, the projected image on electron micrographs hinders interpretation and obscures interactions $[3,4]$. To enhance our ability to interpret dislocation particle interactions, we employ time-resolved straining experiments, conventional $|\bar{g} \cdot \bar{b}|=0$ analysis, along with tomographic reconstructions from a series of bright-field and weak-beam dark-field diffraction contrast micrographs. Combining these approaches provides insight to the temporal and spatial evolution of the dislocation structures preliminary results are presented and discussed.

Time resolved studies of dislocation particle interactions at elevated temperatures have shown that low angle grain boundaries can pass around an $\mathrm{Al}_{3} \mathrm{Sc}$ particle in an aluminum matrix with only about $30 \%$ reduction in the boundary velocity. Interfacial contrast changes during the interaction also suggested the bypass process involved interaction between the lattice and interfacial dislocations. To better understand how dislocation arrays interact with such particles, a 3D tomographic reconstruction was made from weak-beam dark-field micrographs acquired every one degree of tilt over a range of 60 degrees. Figure 1 shows a series of weak-beam dark-field micrographs of such an interaction as seen from different tilts. The wrapping of an $a / 2[110]$ screw dislocation around the particle can now be followed by rotating the tomogram (not shown), as can the attachment of some boundary dislocations to the particle. Similarly, understanding the dislocation arrangements which are observed to be attached and adjacent to particles following bypass interactions with multiple dislocations is difficult from projected images alone. Figure 2a shows matrix and interfacial dislocations around and adjacent to an $\mathrm{Al}_{3} \mathrm{Sc}$ particle and the corresponding tomogram in Figure $2 \mathrm{~b}$ and $\mathrm{c}$. For the $3 \mathrm{D}$ reconstruction, images were acquired every one degree over a range of nearly 60 degrees about two orthogonal 200-type diffraction vectors. The loop seen away from the particle is known from the dynamic experiments to be associated with release of a prismatic loop to remove debris attached to the particle. Two other partial loops, extending from the particle appear to be in the process of cross-slipping to a more favorable plane. Line dislocations as well as the network of interfacial dislocations can be seen following the contour of the particle. The relevance of these observations to our understanding of dislocation-particle interface interactions will be 
discussed. The authors gratefully acknowledge the Department of Energy [5] for funding support and the Center for Microanalysis of Materials, University of Illinois, where this work was carried out.

\section{REFERENCES}

[1] Y. Xiang, D. J. Srolovitz. Philosophical Magazine. 86 (2006) 3937.

[2] Y. Xiang et al. Acta Materialia. 52 (2004) 1745.

[3] B. G. Clark et al. Journal of Materials Research. 20 (2005) 1792.

[4] L. M. Dougherty et al. Acta Materialia. 51 (2003) 4367.

[5] Funding was provided through contract DOE DE-FG02-07ER.

[6] Ian M. Robertson Research Group: http://robertson.mse.uiuc.edu/
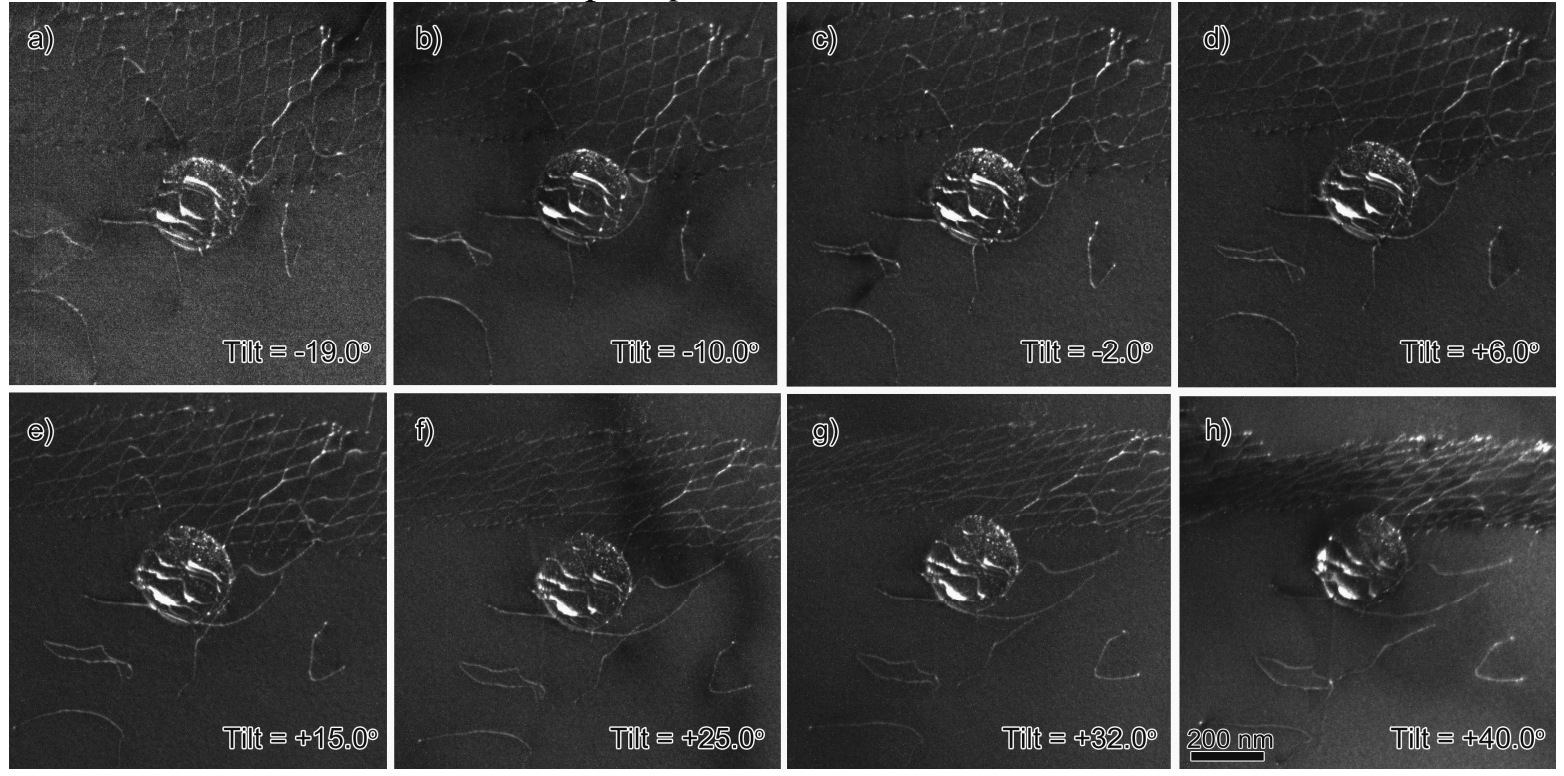

FIG. 1. Weak-beam dark-field micrographs of a $\mathrm{Al}_{3} \mathrm{Sc}$ precipitate interacting with a twist boundary at different degrees of sample tilt. Note the changes in dislocation configuration close to the precipitate as tilt angle is increased.
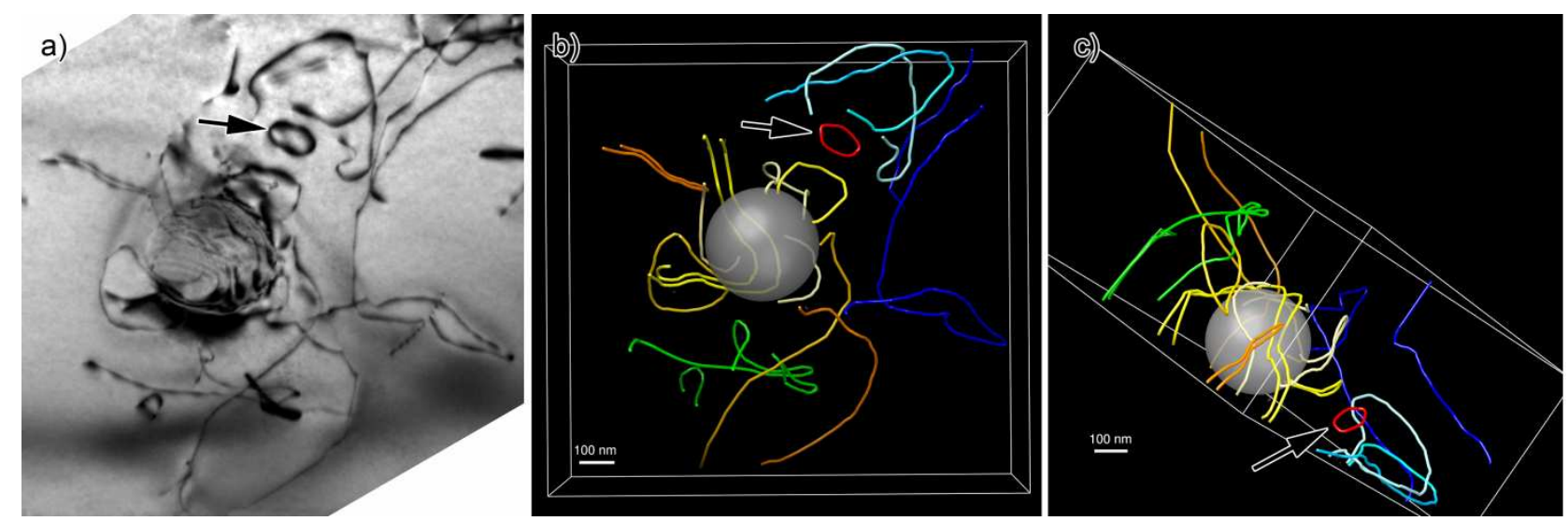

FIG. 2. (a) $\mathrm{An}_{3} \mathrm{Al}_{3} \mathrm{Sc}$ precipitate with arrow indicating a post-interaction loop and (b) the same view in a dislocationtraced tomogram. In an alternate view, (c), the partial loops extending from the particle-matrix interface are more clearly visualized. Colored versions of figures and animations may be viewed in the online proceedings, or at our website [6]. 